\title{
An update on the AM CVn family
}

\section{Elmé Breedt*}

University of Warwick

E-mail: E.Breedt@warwick.ac.uk

I present a brief overview of the known population of AM CVn stars, and compare their properties with the more common hydrogen-rich cataclysmic variables. I focus specifically on the impact of photometric transient surveys on our knowledge of these binaries - both in terms of the discovery of new systems as well as the insights gained from follow-up observations of transient events.

The Golden Age of Cataclysmic Variables and Related Objects - III

7-12 September 2015

Palermo, Italy

${ }^{*}$ Speaker. 


\section{Introduction}

Accreting white dwarf binaries account for a large fraction of the transient events observed by wide-field photometric surveys such as the Catalina Real-time Transient Survey (CRTS), the Palomar Transient Factory (PTF) and the All-Sky Automated Search for Supernovae (ASAS-SN). While most of these are due to dwarf nova outbursts of hydrogen-rich cataclysmic variables (CVs), a number of new AMCVn stars have been discovered this way as well (e.g. [1, 2, 3]).

The AM CVn stars are closely related to the more common cataclysmic variables. They consist of white dwarfs accreting from lower mass companions through Roche lobe overflow, but their optical spectra are hydrogen deficient. ${ }^{1}$ They are very compact binaries, with observed orbital periods that range between $5-65 \mathrm{~min}$, well below the period minimum observed in cataclysmic variables $\left(P_{\min } \sim 80 \mathrm{~min}\right.$; [4]). Such short orbital periods are possible only if the donor star is an evolved star as well. This is best illustrated by the period-density relation for Roche lobe filling stars (e.g. [8]),

$$
\langle\rho\rangle \simeq 107 P_{\text {orb }}^{-2} \mathrm{~g} \mathrm{~cm}^{-3}
$$

where $\langle\rho\rangle$ is the average density of the donor and $P_{\text {orb }}$ the binary orbital period. For the short periods observed in AMCVn stars, the average densities required greatly exceed the typical average densities of main sequence stars. UV spectra of AMCVn stars show enhanced abundances of nitrogen compared to carbon (as measured by the NV/CiV ratio; [9]), which lends further support to the notion of an evolved donor. The interpretation is that the carbon has been processed into nitrogen via the CNO-process.

The donor star plays a key role in the evolution of an AMCVn star. In most cases, two phases of common envelope evolution are required to reach the observed short orbital periods. It is the nature of the donor star when the binary leaves the second common envelope which defines the three so-far accepted channels for AM CVn evolution: $(i)$ If the donor star is another white dwarf, gravitational radiation will bring the two stars closer together, until the lower mass white dwarf fills its Roche lobe at an orbital period of 2-3 min and starts transferring mass. Mass loss from the degenerate donor increases its radius, so the orbital separation increase and the (now semi-detached) binary evolves to longer periods $[10,11]$. At the shortest periods the mass transfer has to occur as direct impact, rather than through an accretion disc, because of the small separation between the two stars. The mass transfer is usually assumed to be stable if the mass ratio $q=M_{2} / M_{1} \lesssim 2 / 3$, but at these small separations, the absence of a disc to redistribute angular momentum as well as the finite size of the accreting white dwarf may destabilise the mass transfer, causing the binary to merge [12]. The binary will only survive as a mass transferring AMCVn star for a small range of parameters, unless the spin of the accretor can be synchronised with the binary orbit over short timescales to stabilise the system [12,13]. Nevertheless, this channel is thought to be responsible for most of the AMCVn population [14]. (ii) If instead the donor is a helium burning star at the onset of mass transfer, it will evolve to a minimum period of $\sim 10$ min before the donor becomes

\footnotetext{
${ }^{1}$ The shortest period binary of this class, HMCnc, appears to have some residual hydrogen [5], though is usually counted among the AMCVn stars. Its exact evolutionary state is still debated, but e.g. [6] show that the system may be explained as a double degenerate binary with an extremely low mass (ELM) white dwarf donor. In this case, the accreted hydrogen originates from the thick envelope of the ELM white dwarf, and the mass transfer is stabilised by its slow response to mass loss. See [7] for a recent overview.
} 
the degenerate and starts to evolve to longer periods as in the double white dwarf case $[15,16]$. (iii) In the third scenario, the binary evolves as a cataclysmic variable, through only one common envelope phase. If the mass ratio is such that the donor star has already started core helium burning by the time mass transfer starts, the binary can reach a shorter period than is possible for the normal $P_{\text {min }}$ of cataclysmic variables $[17,18]$. Throughout its evolution, the binary will get increasingly helium rich and some may exhaust all their hydrogen to become AMCVn stars. Due to the long evolutionary timescale required, this channel is thought to contribute a negligible fraction (much less than 2 per cent) to the total AM CVn population [14, 19].

Detailed reviews of the observational and theoretical work on AMCVn stars have been presented by e.g. [20, 21, 22, 23, 24, 25]. Here I will highlight recent results from transient surveys and compare the optical properties of the AMCVn stars to the hydrogen-rich cataclysmic variables.

\section{Accretion rate evolution}

As a result of the strong dependence of gravitational wave radiation on orbital separation, the mass transfer rate $\dot{m}_{2}$ in AMCVn stars is a steeply decreasing function of orbital period,

$$
\dot{m}_{2} \simeq 5 \times 10^{-9} M_{\odot} \mathrm{yr}^{-1}\left(P_{\text {orb }} / 1000 \mathrm{~s}\right)^{-5.2}
$$

(e.g. $[26,27,8]$ ), with the exact value of the exponent depending on the structure of the donor star and the masses of the binary components. Observationally, this strong accretion rate dependence divides the population into four groups: $(i)$ very compact $\left(P_{\text {orb }} \lesssim 10 \mathrm{~min}\right.$ ) double degenerate binaries, accreting through direct impact, (ii) young, high accretion rate systems, accreting through an optically thick disc. They are in a permanently bright state, similar to the novalike CVs, and have periods $10 \lesssim P_{\text {orb }} \lesssim 20 \mathrm{~min}$ ). (iii) systems at intermediate mass transfer rates, which display outbursts similar to CV dwarf novae, and (iv) faint, long period ( $P_{\text {orb }} \gtrsim 40 \mathrm{~min}$ ) systems for which the continuum emission is dominated by the white dwarf, the optically thin disc produce strong emission lines and no outbursts are observed. These are the oldest, lowest luminosity AMCVn stars and hence the most difficult to discover, but it is also where we expect to find the majority of the population [28].

There is a strong decrease in the UV luminosity of AM CVn stars with increasing orbital period [29], due to the strong contribution of the bright accretion disc at short orbital periods, and the drop in accretion rate as they evolve to longer periods.

[30] and [27] showed that the helium discs of AMCVn stars are subject to a thermal-viscous instability, similar to CVs. They applied the disc instability model to show that outbursts are expected for orbital periods between $20 \lesssim P_{\text {orb }} \lesssim 45 \mathrm{~min}$ in helium binaries, with some adjustments made to the model to account for the difference in composition and the small size of the disc.

\section{Space density estimates}

There are currently 49 known AMCVn stars: 41 listed in [31], one discovered as part of a search for white dwarfs in SDSS [32], six recent transient discoveries shown in Table 1 and one reported by M. Motsoaledi (this proceedings). 38 of these have measured orbital periods, and their period distribution is shown in Fig. 1. They were discovered in a variety of ways, and the overall 

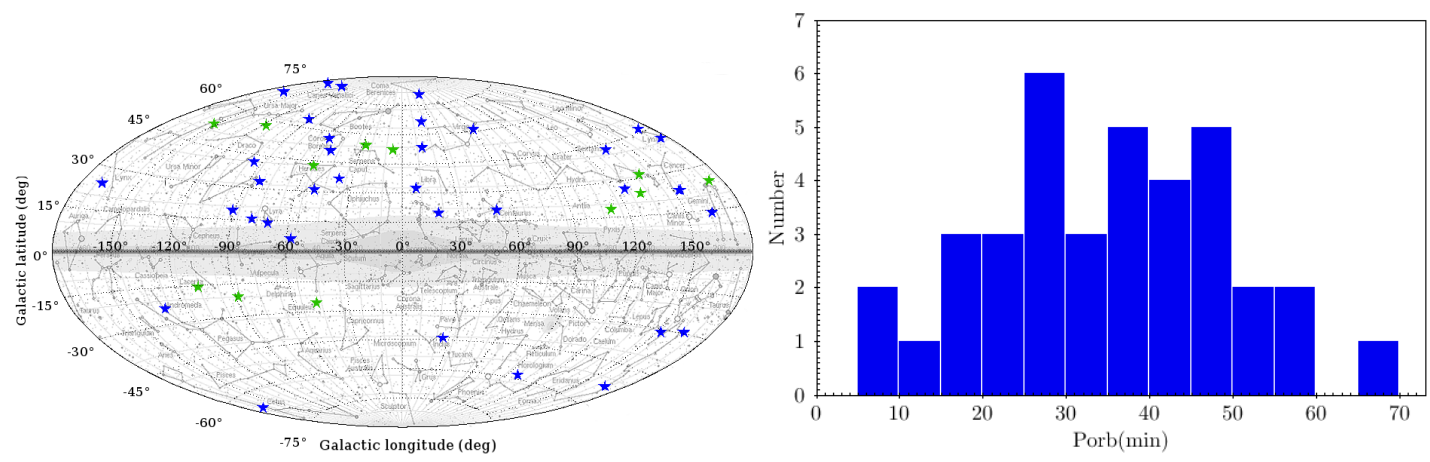

Figure 1: Left: The location of the currently known AMCVn stars in Galactic coordinates. The blue star symbol indicates that the orbital period of the binary is known. Those plotted in green have no reliable period measurement yet. Right: The orbital period distribution of the 38 AMCVn stars with known orbital periods.

population is still biased towards the brightest (high accretion rate) and most variable (outburst) systems.

It is only since the start of large scale surveys that well-defined, albeit small, samples of AMCVn stars could be identified to make reliable estimates of their space density. The first such estimate was made based on the six AMCVns which were serendipitously targeted for spectroscopy by the SDSS [33]. Taking into account the spectroscopic completeness of the survey and comparing it to the expectation from population synthesis, the space density was estimated to be $1-3 \times 10^{-6} \mathrm{pc}^{-3}$, an order of magnitude lower than even the 'pessimistic' prediction from population synthesis models [28].

The six systems were noted to fall in a small region of $u-g, g-r$ colour space. The SDSS spectroscopy selected only $\sim 20 \%$ of targets in this colour region for spectroscopy, so a dedicated survey was carried out to spectroscopically identify the remaining $\sim 2000$ objects $[34,35,36]$.

More than $70 \%$ of candidates with $g<20.5$ have been followed up so far, and to a limit of $g<19.0$ the follow-up is complete (Fig. 2). Seven new AMCVn binaries were discovered as a result of this targeted follow-up. Scaling from the observed space density estimate of [33], 11 new AMCVn binaries brighter than $g<19.0$ were expected to be found, however, only four of the seven discoveries match this limit.

The lower than expected recovery of AMCVn stars raises a number of questions. It implies that the space density is another factor of five lower than previously measured, at $(5 \pm 3) \times$ $10^{-7} \mathrm{pc}^{-3}$. Is the population fainter than we thought, with many more systems to be discovered $g>19.0$ ? Are we biased by the colour selection? Is the birth rate of AMCVn stars lower than we thought, with more binaries merging due to unstable mass transfer? Observations are ongoing to uncover the AMCVn stars in the faint magnitude bins, but a detailed analysis of the potential biases of the follow-up sample suggest that the low space density is a robust result [36, 37]. This sets the space density of AMCVn stars as about an order of magnitude lower than the non-magnetic hydrogen-rich cataclysmic variables [38]. 


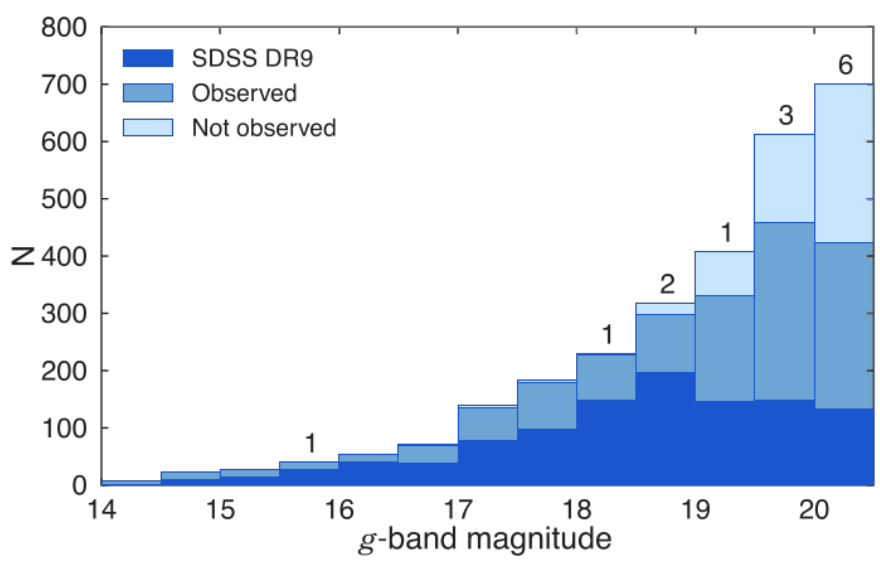

Figure 2: Results of the spectroscopic follow-up in the SDSS search for AM CVn stars. The dark blue bars indicate the systems in the surveyed colour region which have SDSS spectra, the lighter bars indicate the spectroscopy carried out as part of the targeted AMCVn search, and the lightest blue bars indicate those for which spectroscopy is still to be obtained. The follow-up observations are essentially complete down to $g<19$, so, the revised space density estimate is based on those systems only. The number above the bars are the number of known AMCVn stars in that bin. Figure from [36].

\section{Insights from transient follow-up}

Time series spectroscopy has so far been the major tool for characterising the AMCVn population at optical wavelengths. Spectroscopy provides insights into the disc/donor composition, and the strong emission lines are key to the discovery of especially the faint, long period systems. Dynamical mass measurements and Doppler tomography revealed the extreme mass ratios of these binaries, as well as the dynamics of the accretion flow and accurate orbital periods. (e.g. [34, 39, 40, 41, 42]). Using high resolution phase resolved spectroscopy, the narrow 'central spike' emission feature in GPCom was shown to originate from the high electron density environment near the accreting white dwarf. The radial velocity of the spike traces the motion of the accretor, constraining its velocity amplitude to $K_{1}=11.7 \mathrm{~km} \mathrm{~s}^{-1}$ [39, 43].

Wide-field transient surveys have proved to be an efficient way of discovering new outbursting AMCVn stars (Fig. 3). Many outbursting AMCVns display superhumps during superoutbursts, (e.g. [44, 45, 46, 47, 48]), so with rapid follow-up it is possible to measure the periods of even very faint systems with time series photometry. Transient surveys also have the advantage that targets are not colour-selected, although it does limit the discovery space to AMCVn systems with unstable accretion discs. 17/49 of the known population was discovered in transient surveys, with another seven discovered directly as a result of their variability. Not only are these surveys contributing a large number of new systems to the population, but follow-up observations have led to important new insights, which I will briefly summarise in the next few sections.

\subsection{Recently discovered systems}

Follow-up photometry of ASAS-SN transients, mainly by citizen science observers (e.g. AAVSO ${ }^{2}$

\footnotetext{
${ }^{2}$ American Association of Variable Star Observers, http://www.aavso.org
} 


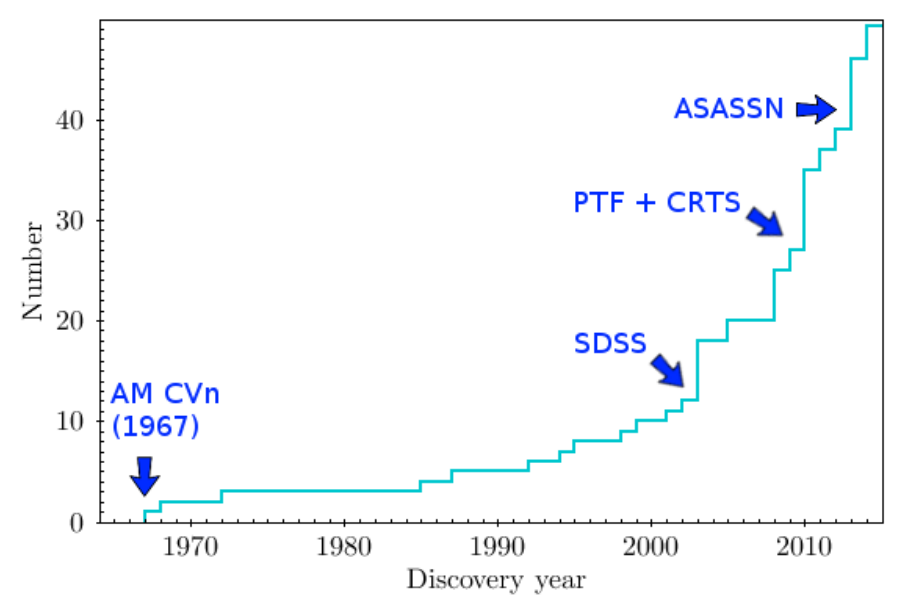

Figure 3: The number of known AM CVn stars have greatly increased in the past few years due to dedicated searches among colour-selected objects in SDSS and follow-up of transient events. The start of each of the surveys is marked on the cumulative distribution.

and $v{ }^{3}{ }^{3}$ ), have revealed six new AMCVns in the past two years (Table 1). The observations highlight the importance of rapid follow-up of outbursting CVs and AMCVn stars, as well as the valuable contribution made by citizen science observers to the field. Period estimates (from superhumps in most cases) could be made for most of the new discoveries because of the rapid response to the transient alerts. In addition, several new or unusual properties of AMCVn stars have been discovered from these observations, such as the very frequently outbursting ASASSN-14cc [3]. It is observed to spend $\sim 60 \%$ of its time in a high accretion state, with outbursts every few days. This behaviour is not well understood within the standard disc instability model, but it is similar to the frequently outbursting RZLMi stars among CVs [49, 50]. The observation of such frequent outbursts in a helium binary provides a strong link between the physical processes operating in the accretion discs of hydrogen and helium binaries.

Table 1 show the periods of these recently discovered systems. Along with the identification spectra shown in Fig. 4, these observations confirm their nature as AMCVn stars.

\subsection{Helium accretion discs}

Several other observations also support the modified disc instability model for AMCVn outbursts. For example, post-outburst monitoring of the ASAS-SN AMCVns revealed that many display multiple short echo outbursts or rebrightenings following a superoutburst (Fig. 5), as is often observed in CVs with extreme mass ratios $[55,56]$. ASASSN-14ei is worth noting as an extreme example of this phenomenon. It had 12 rebrightening episodes in the eight months after its discovery - more than have been observed in any other system. It also had another short outburst only five months later, but it is unclear whether this is an independent outburst or another rebrightening. (See K. Isogai, this proceedings).

More detailed monitoring also mean that the outbursts themselves are observed in greater detail. Features like the pre-outburst dip commonly observed in the superoutburst light curves of

\footnotetext{
${ }^{3}$ Variable Star Network, http://www.kusastro.kyoto-u.ac.jp/vsnet
} 


\begin{tabular}{ccccc}
\hline Target & RA \& Dec & Period [min] & mag & Ref \\
\hline ASASSN-14cc & $21: 39: 48.24-59: 59: 32.4$ & $22.5(\mathrm{sh} ?)$ & 20.0 & {$[3]$} \\
ASASSN-15kf & $15: 38: 38.24-30: 35: 49.7$ & $27.67(\mathrm{sh})$ & 19.4 & {$[51]$} \\
ASASSN-14mv & $07: 13: 27.28+20: 55: 53.4$ & $40.8(\mathrm{sh})$ & 17.3 & {$[52]$} \\
ASASSN-14ei & $02: 55: 33.39-47: 50: 42.0$ & $43(\mathrm{sh})$ & 18.0 & {$[53]$} \\
Gaia14aae $^{\dagger}$ & $16: 11: 33.97+63: 08: 31.8$ & $49.7(\mathrm{orb})$ & 18.0 & {$[62]$} \\
ASASSN-14fv & $23: 29: 55.13+44: 56: 14.4$ & unknown & 20.5 & {$[54]$} \\
\hline
\end{tabular}

${ }^{\dagger}$ Also known as ASASSN-14cn. Orbital period from eclipses.

Table 1: Recently discovered AMCVn systems from follow-up of transients detected by the ASAS-SN survey.

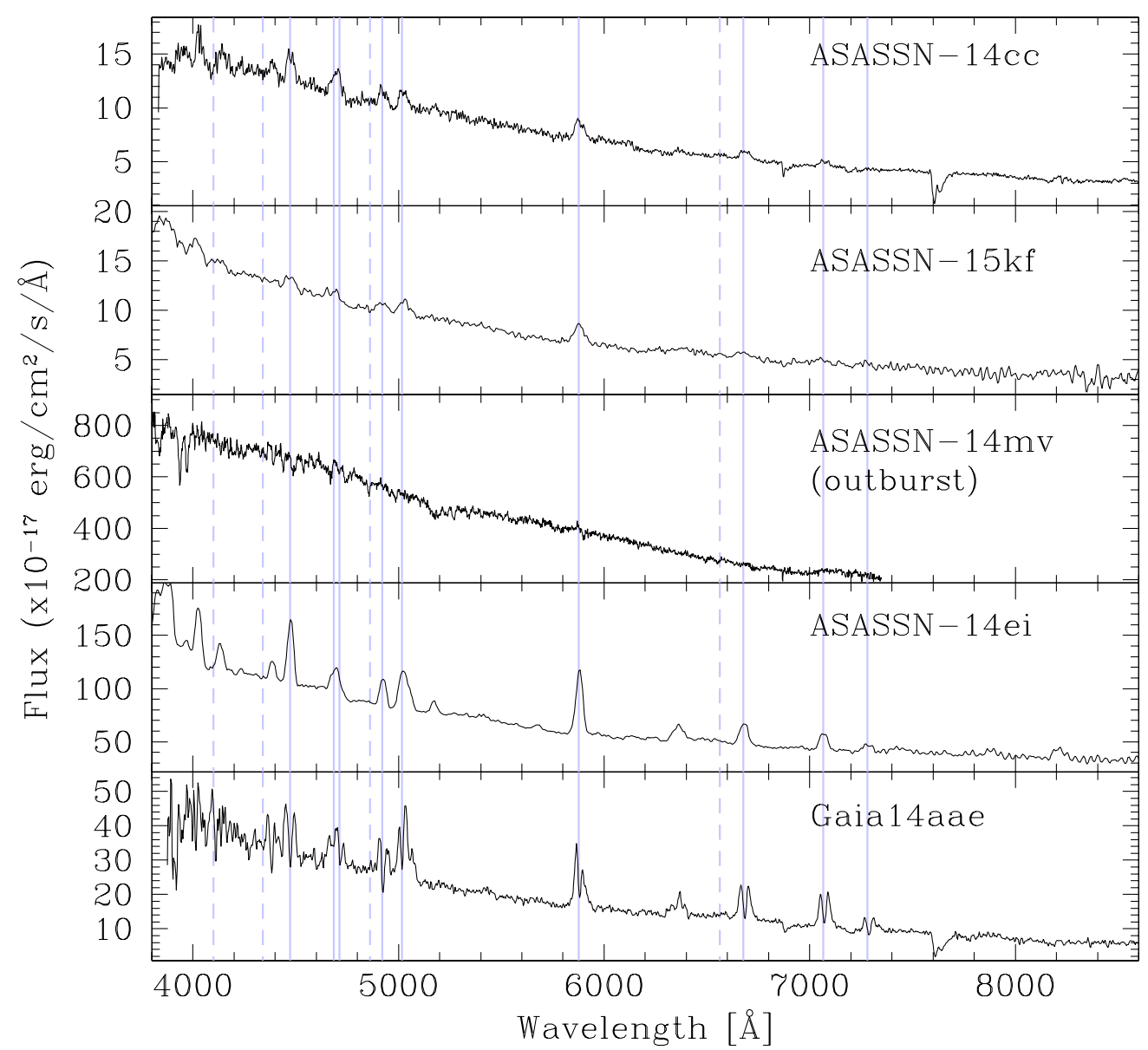

Figure 4: Identification spectroscopy of recently discovered AMCVn stars by the ASAS-SN survey. The vertical lines indicate the wavelengths of $\mathrm{HeI}$ and $\mathrm{H}$ lines, plotted as solid and dashed lines respectively. The spectrum of ASASSN-14mv was taken while the system was still in outburst. It is not possible from this spectrum to rule out the presence of hydrogen to confirm it as an AMCVn system. (The spectrum of Gaia14aae is from [62]; the others from Breedt et al, in preparation.) 


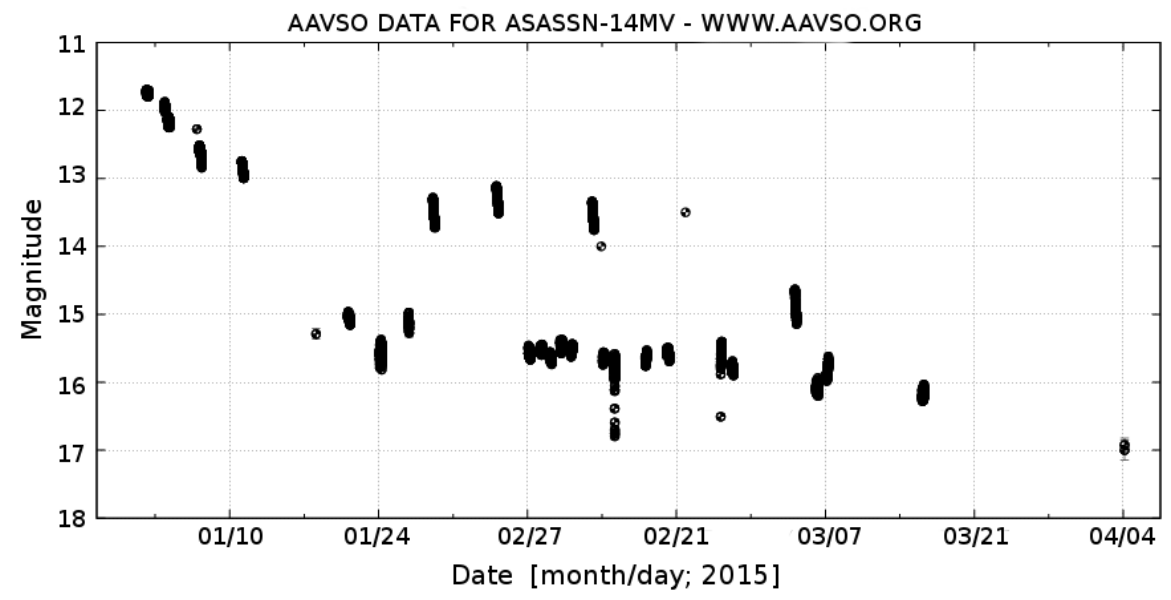

Figure 5: Echo outbursts in the light curve of ASASSN-14mv, following the superoutburst that caused its discovery. Data from the American Association of Variable Star observers, http://www.aavso.org.

CVs, are now seen to be a feature of AMCVn outbursts as well [57, 1]. This feature results from the outward spreading of the heating front through the disc when the outburst starts - the superoutburst is triggered only if there is sufficient material stored in the outer disc [58, 59]. It is an intrinsically disc-based phenomenon, and it lends strong support to the helium disc instability model.

\subsection{Long term photometric behaviour}

Two and a half years of monitoring a number of AMCVn stars [57] showed that the known population broadly agrees with the expectation from the helium disc instability model that outbursts should be observed in systems with orbital periods between $20 \leq P_{\text {orb }} \leq 45$ min. However, transient surveys are increasingly finding AMCVns in outburst with orbital periods longer than $45 \mathrm{~min}$, e.g. CRTS J045020 $-093113\left(P_{\text {orb }}=47.28 \mathrm{~min}\right)$ [60], SDSS J090221.35+381941.9 $\left(P_{\text {orb }}=48.31 \mathrm{~min}\right)$ [61] and Gaia14aae $\left(P_{\text {orb }}=49.71 \mathrm{~min}\right)$ [62].

[31] used the combined light curves from several wide field photometric surveys to construct 6-9 year light curves for all the known AMCVn stars. They investigated the outburst properties of the sample and showed that the time between successive outbursts increase as a power law with orbital period. The outburst interval can be taken as a proxy for the mass transfer rate from the donor, so this is consistent with the expectation that these binaries will have a strongly decreasing $\dot{m}_{2}$ with orbital period (section 2). Although their relation is derived from systems with $22 \leq P_{\text {orb }} \leq$ 37 min for which reliable outburst data is available, the new long period outbursting systems are consistent with this relation and the 9 year length of the light curves. At their measured orbital periods, only one outburst is expected in 9-12 years.

[26] applied the helium disc instability model of [27] to this data and showed that the mass of the accretor also plays an important role. The rate at which the white dwarf accretes material scales inversely with its mass, $\dot{m}_{1} \propto M_{1}^{-2.5}$, so it is possible that these long period outbursting AMCVns have higher primary masses, resulting in a steeper $\dot{m}_{2}-P_{\text {orb }}$ relation.

\subsection{Eclipsing AM CVn stars}

Given the importance of the structures of both the donor and the accretor to the evolution of 
an AMCVn star, mass measurements and parameter studies are hugely important to gain a detailed understanding of these binaries and their evolutionary processes. The best and most reliable way to measure the parameters of the component stars is through light curve modelling of eclipsing systems. Three eclipsing AMCVn binaries are now known: SDSS J092638.71+362402.4 (white dwarf partially eclipsed, [45]), PTF1 J191905.19+481506.2 (only the edge of the disc and the bright spot eclipsed, so not useful for parameter studies, [63]) and Gaia14aae (the first fully eclipsing AM CVn system, [62]).

Although SDSS J092638.71+362402.4 was discovered from spectroscopy and not directly as a result of an outburst, several outbursts have been observed in this system. As both a orbital period (from eclipses) and a superhump period (in outburst) have been measured with high precision, it serves as an important calibrator of the empirical relationship between the superhump period excess $\varepsilon=\left(P_{\mathrm{sh}}-P_{\text {orb }}\right) / P_{\text {orb }}$ and the mass ratio $q[45,64]$. Light curve eclipse modelling shows that the donor in this binary is larger than a zero-temperature white dwarf would be, so it must still contain significant thermal energy.

Follow-up of the Gaia transients have recently revealed that Gaia14aae is the first AMCVn binary in which the accretor is fully eclipsed [62], making it the ideal system for parameter studies. An analysis of its light curve gives a mass ratio $q=0.019$, donor mass $M_{2}=0.015 M_{\odot}$ and accretor mass $M_{1}=0.79 M_{\odot}$, assuming an edge-on view of the binary. Gaia14aae is also the longest period AMCVn for which outbursts have been observed, and its high primary mass supports the conclusion of [26] that higher mass white dwarfs will have longer outburst intervals. The donor mass is consistent with a zero entropy donor at the observed $P_{\text {orb }}=49.7 \mathrm{~min}$ [65], but as the inclination is not yet precisely measured, this represents a minimum mass of the donor. Ongoing high-speed photometry will allow us to measure the eclipse of the faint bright spot with greater precision in the future, from which we will be able to measure the inclination and improve the component mass measurements.

\subsection{Sub-period minimum CVs}

Transient surveys are also starting to reveal potential progenitors of AMCVn stars, particularly those that evolve along the often-dismissed evolved CV channel (see section 1). These are binaries which have periods and mass ratios similar to the long period AM CVn binaries, but still have some residual hydrogen. Their optical spectra display enhanced He/H ratios compared to normal CVs, which is interpreted as hydrogen burning prior to the onset of mass transfer, and then a loss of the residual hydrogen through further evolution and mass transfer.

There are now 10 systems known which have hydrogen in their spectra, but periods below $P_{\text {min }}$ $[66,2]$. Not all these systems will evolve to become AMCVn stars (some will simply reach their minimum orbital periods before all the hydrogen is exhausted), but it is clear that this formation channel can produce binaries with a wide range of parameters. Indeed, several CVs with evolved donors are known as well (e.g. [67, 68, 69]). So far at least two of these sub- $P_{\min }$ binaries show spectroscopic properties and mass ratios that are very similar to the known AMCVn stars [66, 70], which suggest that they may be true AMCVn progenitors. It is also possible that some of the long period outbursting AMCVn stars are systems which have evolved along this channel. If so, their donor stars should be significantly hotter than that of a system which has evolved along the double degenerate or helium star route to the same orbital period. Further studies of the sub- $P_{\min } \mathrm{CVs}$ 
will help us to understand the significance of the $\mathrm{CV}$ channel for AMCVn formation as well as its impact on the $\mathrm{CV}$ population in general.

\section{Final comments and future work}

- Variability surveys have revealed 17 new AMCVn stars in the past 5 years - more than a third of the known population. Future surveys like Gaia and LSST promise to uncover many more, but follow-up observations are essential to characterise the binaries. Since many of the systems discovered by transient surveys are very faint in quiescence and not suitable for phase resolved spectroscopy, rapid follow-up is needed to look for superhumps in the light curves to measure their periods. Citizen science observers have made a huge contribution to $\mathrm{CV}$ and AMCVn science in the past, and continue to enrich this field with their contributions.

- As most surveys are carried out at high Galactic latitudes, this is where most of the currently known AMCVn stars are found (Fig. 1). In order to uncover the large number of systems expected at low Galactic latitudes [28] and also systems with smaller variability amplitudes, specific searches such as the OmegaWhite Survey [71], are underway.

- The nature of the donor star plays a central role in the evolution of these binaries, but in none of these systems has the donor star ever been detected directly. So far, the composition of the donor star can only be inferred though observations of the accretion disc, and theoretical efforts are being made to model the helium accretion discs as a probe of the donor stars [72].

- Space-based gravitational wave detectors, such as eLISA, are expected to detect hundreds of AM CVn stars in the low frequency gravitational wave sky [37]. In particular, it will be sensitive to the shortest period binaries which are hidden from variability or emission line surveys carried out so far. As the only sources for which electromagnetic observations are currently available, they will serve as important gravitational wave verification and calibration sources [37].

\section{Acknowledgements}

I would like to thank Danny Steeghs for helpful discussions and the conference organisers for the invitation to present this review.

\section{References}

[1] Levitan, D. et al, Five new outbursting AMCVn systems discovered by the Palomar Transient Factory, MNRAS, 430, 996 (2013)

[2] Breedt, E. et al, 1000 cataclysmic variables from the Catalina Real-time Transient Survey, MNRAS, 443, 3174 (2014)

[3] Kato, T. et al, ASASSN-14cc: A likely helium analog of RZ Leonis Minoris, PASJ, 67L, 2 (2015)

[4] Gänsicke, B. T. et al, SDSS unveils a population of intrinsically faint cataclysmic variables at the minimum orbital period, MNRAS, 397, 2170 (2009) 
[5] Reinsch, K. et al, Spectral Analysis and Constraints on the Nature of the Ultra-Compact Binary RX J0806.3+1527, in proceedings of The 15th European Workshop on White Dwarfs, ASPC, 372, 419 (2007)

[6] Kaplan, D. et al, Orbital Evolution of Compact White Dwarf Binaries, ApJ, 758, 64 (2012)

[7] Esposito, P. et al, Swift X-ray and ultraviolet observations of the shortest orbital period double-degenerate system RX J0806.3+1527 (HM Cnc), A\&A, 561A, 117 (2012)

[8] Warner, B. Cataclysmic variable stars., Cambridge Astrophysics Series, Cambridge, 2003

[9] Gänsicke, B. T. et al, Anomalous Ultraviolet Line Flux Ratios in the Cataclysmic Variables $1 R X S$ J232953.9+062814, CE 315, BZ Ursae Majoris, and EY Cygni, Observed with the Hubble Space Telescope Space Telescope Imaging Spectrograph, ApJ, 594, 443 (2003)

[10] Paczýnski, B., Gravitational Waves and the Evolution of Close Binaries, Acta Astron., 17, 287 (1967)

[11] Faulkner, J. et al, Ultrashort-Period Binaries. II. HZ 29 (=AM CVn): a Double-White Semidetached Postcataclysmic Nova?, ApJ, 175, L79 (1972)

[12] Marsh, T. R. et al, Mass transfer between double white dwarfs, MNRAS, 350, 113 (2004)

[13] Kilic, M. et al, Massive double white dwarfs and the AM CVn birthrate, MNRAS, 460, 4176 (2016)

[14] Nelemans, G. et al, The chemical composition of donors in AM CVn stars and ultracompact X-ray binaries: observational tests of their formation, MNRAS, 401, 1347 (2010)

[15] Savonije, G. J. et al, The minimum orbital period for ultra-compact binaries with helium burning secondaries, $A \& A, \mathbf{1 5 5}, 51$ (1986)

[16] Iben, I. \& Tutukov, A. V., Evolutionary scenarios for intermediate-mass stars in close binaries, ApJ, 313, 727 (1987)

[17] Sienkiewicz, R., The minimum period of hydrogen-deficient cataclysmic binaries, Acta Astron., 34, 325 (1984)

[18] Podsiadlowski, P. et al, Cataclysmic variables with evolved secondaries and the progenitors of AM CVn stars, MNRAS, 340, 1214 (2003)

[19] Piersanti, L. et al, He-accreting WDs: AM CVn stars with WD donors, MNRAS, 452, 2897 (2015)

[20] Ulla, A. The AM CVn Systems: A Bibliographic Search for Clues to Their Cataclysmic Nature, SSRv, 67, 241 (1994)

[21] Nelemans, G., AM CVn stars, in proceedings of The Astrophysics of Cataclysmic Variables and Related Objects, ASPC, 330, 27 (2005)

[22] Ramsay, G. et al, XMM-Newton observations of AM CVn binaries, A\&A, 440, 675 (2005)

[23] Ramsay, G., Recent Observational Progress in AM CVn Binaries, in proceedings of The 15th European Workshop on White Dwarfs, ASPC, 372, 425 (2007)

[24] Solheim, J.-E., AM CVn Stars: Status and Challenges, PASP, 1221133 (2010)

[25] Warner, B. Aspects of observations and evolution of AM CVn stars, MmSAI, 86, 129 (2015)

[26] Cannizzo, J. K. \& Nelemans, G., Constraining the Physics of AM Canum Venaticorum Systems with the Accretion Disk Instability Model, ApJ, 80319 (2015)

[27] Kotko, I. et al, Models of AM Canum Venaticorum star outbursts, A\&A, 544, A13 (2012) 
[28] Nelemans, G. et al, Population synthesis for double white dwarfs II. Semi-detached systems: AM CVn stars, A\&A, 368, 939 (2001)

[29] Ramsay, G. et al, XMM-Newton observations of AM CVn binaries: V396 Hya and SDSS J1240-01, $A \& A, 457,623$ (2006)

[30] Tsugawa, M. \& Osaki, Y., Disc Instbility Model for the AM Canum Venaticorum Stars, PASJ, 49, 75 (1997)

[31] Levitan, D. et al, Long-term photometric behaviour of outbursting AM CVn systems, MNRAS, 446391 (2015)

[32] Kepler, S. O. et al, New white dwarf stars in the Sloan Digital Sky Survey Data Release 10, MNRAS, 4464078 (2015)

[33] Roelofs, G. H. A. et al, The population of AM CVn stars from the Sloan Digital Sky Survey, MNRAS, 382, 685 (2007)

[34] Roelofs, G. H. A. et al, SDSSJ080449.49+161624.8: a peculiar AM CVn star from a colour-selected sample of candidates, MNRAS, 394367 (2009)

[35] Rau, A. et al, A Census of AM CVn Stars: Three New Candidates and One Confirmed 48.3-Minute Binary, ApJ, 708456 (2010)

[36] Carter, P. J. et al, A search for the hidden population of AM CVn binaries in the Sloan Digital Sky Survey, MNRAS, 429, 2143 (2013)

[37] Nissanke, S. et al, Gravitational wave emission from compact Galactic binaries, ApJ, 758, 131 (2012)

[38] Pretorius, M. L. et al, The space density and X-ray luminosity function of non-magnetic cataclysmic variables, MNRAS, 4191442 (2012)

[39] Morales-Rueda, L. et al, New results on GP Com, A\&A, 405, 249 (2003)

[40] Kupfer, T. et al, Orbital periods and accretion disc structure of four AM CVn systems, MNRAS, 432, 2048 (2013)

[41] Carter, P. J. et al, The AM Canum Venaticorum binary SDSS J173047.59+554518.5, MNRAS, 437, 2894 (2014)

[42] Kupfer, T. et al, Phase-resolved spectroscopy and Kepler photometry of the ultracompact AM CVn binary SDSS J190817.07+394036.4, MNRAS, 453, 483 (2015)

[43] Kupfer, T. et al, UVES and X-Shooter spectroscopy of the emission line AM CVn systems GP Com and V396 Hya, MNRAS, 457, 1828 (2016)

[44] Patterson, J. et al, Superhumps in Cataclysmic Binaries. XII. CR Bootis, a Helium Dwarf Nova, PASP, 109, 1100 (1997)

[45] Copperwheat, C. M. et al, SDSS J0926+3624: the shortest period eclipsing binary star, MNRAS, 410, 1113 (2011)

[46] Levitan, D. et al, PTF1 J071912.13+485834.0: An Outbursting AM CVn System Discovered by a Synoptic Survey, ApJ, 739, 68 (2011)

[47] Shears, J. et al, Superhumps and post-outburst rebrightening episodes in the AM CVn star SDSS J012940.05+384210.4, JBAA, 122, 49 (2012)

[48] Isogai, K. et al, Superoutburst of CR Bootis: Estimation of mass ratio of a typical AM CVn star by stage A superhumps, PASJ, 68, 64 (2016) 
[49] Otulakowska-Hypka, M. et al, IX Draconis - a curious ER UMa-type dwarf nova, MNRAS, 429, 868 (2013)

[50] Kato, T. et al, RZ Leonis Minoris bridging between ER Ursae Majoris-type dwarf nova and nova-like system, PASJ, 68, 107 (2016)

[51] Monard, B., ASASSN-15kf: superoutburst of a new AM CVn-type, vsnet-alert 18669 (2015)

[52] Kato, T. et al, ASASSN-14mv: ultrashort-period system with stages A and B, vsnet-alert 18124 (2015)

[53] Prieto, J. L. et al, Follow-up of ASASSN-14ei show He lines in the spectra and Swift/XRT X-ray detection, ATEL\#6475 (2014)

[54] Wagner, R. M. et al, Identification of a New Candidate Outbursting AM CVn System from ASAS-SN, ATEL\#6669 (2014)

[55] Patterson, J. et al, Superhumps in Cataclysmic Binaries. XV. EG Cancri, King of the Echo Outbursts, PASP, 110, 1290 (1998)

[56] Meyer, F. \& Meyer-Hofmeister, E., SU UMa stars: Rebrightenings after superoutburst, PASJ, 67, 52 (2015)

[57] Ramsay, G. et al, The long-term optical behaviour of helium-accreting AM CVn binaries, MNRAS, 419, 2836 (2012)

[58] Cannizzo, J. K. et al, The Kepler Light Curves of V1504 Cygni and V344 Lyrae: A Study of the Outburst Properties, ApJ, 747117 (2012)

[59] Osaki, Y. \& Kato, T., Study of Superoutbursts and Superhumps in SU UMa Stars by the Kepler Light Curves of V344 Lyrae and V1504 Cygni, PASJ, 65, 950 (2013)

[60] Woudt, P. A. et al CSS121123:045020-093113: A WZ Sge-type dwarf nova amongst the AM CVn systems, ATEL\#4726 (2013)

[61] Denisenko, D. et al, SDSS J090221.35+381941.9 outburst!, vsnet-alert 16982 (2014)

[62] Campbell, H. C. et al, Total eclipse of the heart: the AM CVn Gaia14aae/ASSASN-14cn, MNRAS, 452, 1060 (2015)

[63] Levitan, D. et al, PTF1 J191905.19+481506.2 - a Partially Eclipsing AM CVn System Discovered in the Palomar Transient Factory, ApJ, 785, 114 (2014)

[64] Patterson, J. et al, Superhumps in Cataclysmic Binaries. XXV. $q_{\text {crit }}, \varepsilon(q)$, and Mass-Radius, PASP, 117, 1204 (2005)

[65] Deloye, C. et al, The thermal evolution of the donors in AM CVn binaries, MNRAS, 381, 525 (2007)

[66] Breedt, E. et al, CSS100603:112253-111037: a helium-rich dwarf nova with a 65 min orbital period, MNRAS, 425, 2548 (2012)

[67] Thorstensen, J. R. CSS J134052.0+151341: A Cataclysmic Binary Star with a Stripped, Evolved Secondary, PASP, 125, 506 (2013)

[68] Rebassa-Mansergas, A. et al, SDSS J001153.08-064739.2, a Cataclysmic Variable with an Evolved Donor in the Period Gap, ApJ, 790, 28 (2014)

[69] Thorstensen, J. R. ASAS-SN 13cl: A Newly Discovered Cataclysmic Binary with an Anomalously Warm Secondary, PASP, 127, 351 (2015)

[70] Carter, P. J. et al, The helium-rich cataclysmic variable SBSS 1108+574, MNRAS, 431, 372 (2013) 
[71] Macfarlane, S. et al, The OmegaWhite survey for short-period variable stars - I. Overview and first results, MNRAS, 454, 507 (2015)

[72] Gehron, K. et al, Non-LTE spectral analysis of the AM CVn system PTF 09hpk during quiescence, $A \& A, \mathbf{5 6 2 A}, 132$ (2014)

[73] Roelofs, G. H. A. et al, Hubble Space Telescope Parallaxes of AM CVn Stars and Astrophysical Consequences, ApJ, 666, 1174 (2007)

\section{DISCUSSION}

JOE PATTERSON: For the space densities, do you primarily use trigonometric parallaxes, and if so, are they sufficiently accurate and numerous?

ELMÉ BREEDT: There are parallax distances available for only six systems so far: two long period systems and four short period systems. [73] derive a space density using these measurements, but caution that the completeness of that sample is very uncertain. The short period systems are expected to make up only a small fraction of the overall population, and this introduces another large uncertainty into the estimate. Instead what [36] and others did, was to define a more homogeneously selected sample of AMCVn stars from their SDSS colour follow-up, then compare this sample to the predictions of population synthesis models. This way, the models could be calibrated with reliable observational input to derive the overall space density.

NATALY KATYSHEVA: Do you include the systems with hydrogen in the number of AMCVn stars?

ELMÉ BREEDT: No, I consider those progenitors of AMCVn stars, likely evolving along the CV channel suggested by [18]. In the AMCVn count I include only those systems which have no obvious hydrogen in their optical spectra. 